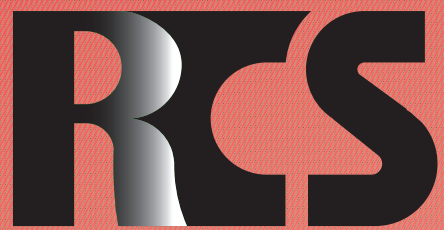

Depósito legal ppi $201502 Z U 4662$

Esta publicación científica en formato digital es continuidad de la revista impresa Depósito Legal: pp $197402 Z U 789$

- ISSN: 1315-9518 • ISSN-E: 2477-9431

Revista de Ciencias Sociales

Universidad del Zulia. Revista de la Facultad de Ciencias Económicas y Sociales Vol. XXVII. No. 2

Abril-Junio 2021

Esta publicación científica en formato digital es continuidad de la revista impresa Depósito Legal: pp $197402 Z$ Z789 ISSN: 1315-9518 


\title{
Mujeres no son personas: Los derechos de las mujeres nahuas de Acaxochitlán, Hidalgo-México
}

\author{
Mendoza Mendoza, Silvia* \\ Durán González, Rosa Elena** \\ Raesfeld, Lydia ${ }^{* * *}$
}

\section{Resumen}

La construcción de persona con derechos en comunidades indígenas nahuas es una condición ajena de las mujeres, el reconocimiento de sus derechos está anclado al patriarca. En el hogar paterno, la subordinación se asimila con la realización de labores domésticas, se naturaliza que el patriarca toma decisiones que afectan a los habitantes del hogar. El objetivo de la investigación es indagar las particularidades de la comunidad nahua sobre los derechos de las personas, especialmente los derechos de las mujeres indígenas en sus comunidades. Se utilizó una metodología mixta en la cual se desarrolló la técnica de grupos de discusión sobre derechos esenciales como la educación, salud e igualdad, se analizaron los testimonios, también se procesaron bases de datos. Como resultado la construcción de persona con derechos y la salvaguarda de estos en comunidades indígenas nahuas, está anclada a la condición masculina, sentido de propiedad y vigilancia, que impone la familia y comunidad. En conclusión, en la vida comunitaria, las autoridades indígenas corresponden a los constructos culturales que asumen como sus pares a los otros hombres. Por tanto, las mujeres indígenas continúan indefensas en el ejercicio de sus derechos humanos porque las instituciones y políticas públicas están permeadas por alianzas patriarcales.

Palabras clave: Mujer indígena; comunidad nahua; derechos; patriarcado; relaciones de poder.

* Doctora en Antropología Social. Profesora e Investigadora del Instituto de Ciencias Sociales y Humanidades de la Universidad Autónoma del estado de Hidalgo, México. E-mail: mendozam_uaeh@yahoo.com.mx iD ORCID: https:// orcid.org/0000-0002-5836-2501

** Doctora en Ciencias de la Educación. Profesora e Investigadora del Instituto de Ciencias Sociales y Humanidades de la Universidad Autónoma del estado de Hidalgo, México. E-mail: rdurango@uaeh.edu.mx iD ORCID: https://orcid. org/0000-0001-8121-5019

*** Doctora en Etnología. Profesora e Investigadora del Instituto de Ciencias Sociales y Humanidades de la Universidad Autónoma del estado de Hidalgo, México. E-mail: raesfeld@uaeh.edu.mx iD ORCID: https://orcid.org/0000-0002$\underline{2589-9628}$ 


\title{
Women are not persons: The rights of nahua women, Acaxochitlan, Hidalgo-México
}

\begin{abstract}
The construction of a person with rights in indigenous nahua communities is a foreign condition of women, the recognition of their rights anchored to the patriarch. In the paternal home, subordination is assimilated through the execution of domestic labor, naturalizing the patriarch making decisions that affect the inhabitants of the home. The purpose of this study is to contribute to the construction of indigenous women as people with rights within their communities. A mixed methodology was used in which the technique of discussion groups on basic human rights such as education, healthcare and equality was developed, analizing testimonies and processing the database. As a result, the construction of a person with rights and the safeguarding of these in Nahua indigenous communities is anchored to the masculine condition, sense of ownership and vigilance, imposed by the family and community. In conclusion, in community life, indigenous authorities correspond to the cultural constructs that they assume as their peers to other men. Therefore, indigenous women continue to be defenseless in the exertion of their human rights because public institutions and policies are permeated by patriarchal alliances.
\end{abstract}

Keywords: Indigenous women; Nahua community; rights; patriarchy; power relationships.

\section{Introducción}

Es reconocido que las mujeres por su condición de género tienen una posición subordinada y desigual ante sus congéneres varones, en pueblos indígenas esa situación se reproduce. Kambel (2012), en su propuesta de guía sobre los derechos de la mujer indígena, resalta la necesidad de hacer evidente la desigualdad e invisibilidad que las indígenas tienen en sus propios contextos y en los análisis de los órganos internacionales involucrados con la defensa de los derechos humanos. Señalar desigualdad femenina indígena como práctica cotidiana en los pueblos, es una afirmación que va en contra de las posturas esencialistas que asumen a las sociedades indígenas como igualitarias y solidarias, en ese sentido, desde el pensamiento feminista indígena se sostiene que el sistema colonial sobre los pueblos indígenas, renovó el patriarcado originario, pero agregando racismo (Cabnal, 2010).

$\mathrm{La}$ evidencia empírica obliga a discutir sobre las formas que hacen posible la permanencia de la cultura indígena bajo un sistema social y cultural que somete a las mujeres y les impide el ejercicio de sus derechos en su condición de personas, en tales contextos, los mandatos culturales prevalecientes reproducen los valores patriarcales aprendidos para su inserción en los procesos que impone la sociedad nacional dominante.

Para este trabajo, los referentes conceptuales asumidos son los derechos humanos de las mujeres indígenas, así como la mirada de género y patriarcado, plausibles para mirar la desigualdad de las mujeres en contextos indígenas con el objetivo de revisar las particularidades que la comunidad Nahua guarda con respecto a los derechos de las personas, especialmente con las mujeres indígenas.

La metodología cuantitativa, contempló el procesamiento de base de datos de institucionales a nivel estatal y municipal, con el fin de mostrar un panorama general de las mujeres y sus condiciones sociales así como culturales. La metodología cualitativa, consistió en el desarrollo de grupos de discusión (Gil, 1992), con 255 mujeres Nahuas en un universo de cinco comunidades: Santa Ana Zacuala, Santiago Tepepa, Los Reyes, San Pedro Tlachichilco 
y San Mateo. Las comunidades concentran el $52 \%$ de la población total del municipio que se cuantificaron en 40.583 habitantes, según datos del Instituto Nacional de Estadística y Geografía (INEGI, 2011); el gobierno municipal reconoce un total de 54 localidades.

La investigación no se propuso establecer representatividad, porque siendo Acaxochitlán un municipio predominantemente rural e indígena con asentamientos poblacionales que se repiten en las comunidades, se priorizó la recuperación de experiencias a partir de ejes temáticos de discusión en grupos integrados por mujeres según su edad cronológica y cultural; las investigadoras estuvieron conscientes sobre las limitaciones de información vertidas, porque el parentesco o vecindad de las interlocutoras se impuso al expresar sus pensamientos y sentires; los inconvenientes de los grupos de discusión en regiones rurales ya fue revisado en término de las consecuencias que supone para las mujeres el expresar públicamente problemas o situaciones que se consideran propios del hogar o la familia (Arias, 2014).

La conformación de los grupos de discusión quedó sujeto a los grupos poblacionales, establecidos desde la normativa que rige la política pública en el estado de Hidalgo-México, por tanto, se conformaron grupos de edad correspondiente a niñas, adolescentes, adultas y adultas mayores, que permitieron profundizar en las condiciones de sus derechos y desigualdades de género. Las mujeres fueron convocadas por la Instancia de la Mujer de Acaxochitlán. Los hallazgos presentan una construcción relacional con los referentes conceptuales, que permiten una mirada analítica y crítica de las desigualdades de género y el patriarcado presente en toda la estructura social comunitaria, pues el objetivo del estudio fue indagar sobre las particularidades que la comunidad Nahua guarda con respecto a los derechos de las personas, especialmente con las mujeres indígenas.

\section{Mandatos de género en el contexto de la comunidad}

Desde un enfoque histórico, Segato (2016) afirma que las estructuras patriarcales remiten a la prehistoria patriarcal de la humanidad; posteriormente las ubica en el mundo aldea o pre-intrusión colonial, donde las relaciones de género se regían por sistemas de creencias, relaciones jerárquicas propias de un sistema comunitario, con prestigios diferenciados para ambos géneros: Ancianos y ancianas tenían presencia y autoridad en la jerarquía, los hombres también ostentaban el poder. Esta estructura la define como un patriarcado de bajo impacto.

Con la colonización ultramarina y posteriormente republicana, las relaciones sociales y de poder del mundo aldea se modificaron de tal forma que el patriarcado de bajo impacto, transitó lentamente hacia un patriarcado de alta intensidad con el olvido de las formas tradicionales de relación y con una evidente "ruptura de los hilos de la memoria de sus miembros" (Segato, 2016, p.110).

Por lo anterior, los mandatos de género son construcciones sociales vigiladas $\mathrm{y}$ sancionadas por los grupos humanos que las significan, también llenan de sentido para distinguir lo femenino y lo masculino; en el sistema patriarcal, tienen el propósito de reproducir el sistema de dominación masculino instalado en todos los ámbitos de la vida. Los mandatos atraviesan a hombres y mujeres, por ejemplo: El matrimonio y la obligada descendencia, en comunidades indígenas los hombres solteros y/o sin hijos son omitidos en las convocatorias de las asambleas generales, ahí la presencia femenina es excepcional y en representación del hombre de la casa.

La asamblea general, es la institución más importante de toma de decisiones para la vida colectiva, la obra pública, la ocupación del espacio y tiempo ritual; por tanto, los varones que incumplen el matrimonio o la procreación de su propia descendencia, son asumidos como menores de edad para discutir y decidir 
sobre la vida colectiva de la comunidad. Mujeres adultas solteras viven en "desgracia" sin marido que las "cuide", ellas permanecen en el hogar paterno como encargadas de sus progenitores o de cualquiera que requiera de cuidados especiales. Al respecto, sostienen Llanes, et al. (2020) que: "Desde siempre, la mujer ha sido la base del hogar y la encargada de la crianza de los hijos" (p.52).

Los privilegios masculinos como la propiedad de la tierra, la toma de decisiones y el prestigio en el espacio público, entre otros, dotaron al patriarcado de contenidos nuevos, como el control y sometimiento de las mujeres al espacio privado, a cuidar del otro, proteger lo pequeño como plantas y animales, así como todo lo relacionado casa adentro. Esta visión androcéntrica de dominación, se fortalece al ser compartida con otros interlocutores que gozan de los mismos privilegios de orden universal, como el reconocimiento económico, político, intelectual, bélico, físico y sexual, que permite perpetuar las desigualdades de género (Segato, 2016).

Esta reflexión abona a la construcción de las mujeres indígenas como sujetas de derechos en sus comunidades, la comprensión de los derechos humanos que, si bien refiere a un reconocimiento de la persona en lo individual, la preservación de los derechos es una condición colectiva, que para el caso de las comunidades indígenas involucra a las familias, comunidades y el gobierno en todos sus niveles (Comisión Nacional de los Derechos Humanos, 2015).

Se parte del principio que las familias y las comunidades indígenas protegen a sus integrantes, pero atendiendo sobre cuál es el sentido de esa protección, porque se vislumbra que las mujeres indígenas no son asumidas como personas en sí, sino a partir de cumplir dos condiciones: Primero, su adscripción a un patriarca que puede ser su abuelo, padre, tío, hermano, marido o hijo; segundo, al cumplimiento del mandato del cuidado para los otros, que inicia con su núcleo familiar y se extiende a cualquier integrante de la comunidad que requiera atención, ello incluye objetos y lugares sagrados comunitarios.

El análisis de la información se realiza con una perspectiva de género, considerando que todos los humanos construyen significados propios sobre la condición femenina o masculina, pero sin ignorar, que desde la conquista española sobre los pueblos originarios iniciado en el siglo XVI se implantó un sistema de pensamiento, que se simbolizó y significó con lógicas patriarcales, monoteístas y racistas que justificaron el trato desigual para las mujeres (Quijano, 2014). En el siglo XVII y XVIII, el discurso igualitario de la modernidad transitó de un orden super jerárquico y desarraigado, a una super inflación de los hombres en el ambiente comunitario. Los hombres en las comunidades, aprendieron a negociar solo con estructuras masculinas mismas que interactuaban con otras alianzas masculinas fuera de la comunidad para incrementar su reconocimiento y prestigio.

\section{Mujeres de comunidades nahuas en Acaxochitlán, Hidalgo}

En el estado de Hidalgo-México, radican tres grupos indígenas según la lengua que hablan: Nahuas, Otomies y Tepehuas. El náhuatl, es la lengua indígena más hablada en la República Mexicana (INEGI, 2016), en el estado de Hidalgo, de un total 2.858.359 habitantes, los hablantes de lengua indígena representan el $21,2 \%$, esto significa que una de cada cinco personas está familiarizada con alguna lengua originaria, pero la lengua náhuatl tiene la mayor cantidad de hablantes, seguida por la lengua otomí (ver Tabla 1). 


\section{Tabla 1}

Estado de Hidalgo, número y porcentaje de población hablante de lengua indígena según la lengua originaria, 2015

\begin{tabular}{|c|c|c|}
\hline Lengua indígena & Número & $\%$ \\
\hline Náhuatl & 363.948 & $60,1 \%$ \\
\hline Otomi & 221.931 & $36,6 \%$ \\
\hline No especificado & 6.744 & $1,1 \%$ \\
\hline Tepehua & 2.900 & $0,5 \%$ \\
\hline Zapoteco & 2.348 & $0,4 \%$ \\
\hline Totonaco & 2.207 & $0,4 \%$ \\
\hline Mixteco & 1.266 & $0,2 \%$ \\
\hline Huasteco & 743 & $0,1 \%$ \\
\hline Mazateco & 615 & $0,1 \%$ \\
\hline Mazahua & 565 & $0,1 \%$ \\
\hline Maya & 537 & $0,1 \%$ \\
\hline Mixe & 413 & $0,1 \%$ \\
\hline Chinanteco & 374 & $0,1 \%$ \\
\hline Tlapaneco & 329 & $0,1 \%$ \\
\hline Tseltal & 275 & $0,0 \%$ \\
\hline Tarasco & 236 & $0,0 \%$ \\
\hline Ch'ol & 169 & $0,0 \%$ \\
\hline Tsotsil & 111 & $0,0 \%$ \\
\hline Zoque & 58 & $0,0 \%$ \\
\hline Triqui & 48 & $0,0 \%$ \\
\hline Amuzgo & 42 & $0,0 \%$ \\
\hline Mam & 33 & $0,0 \%$ \\
\hline Cuicateco & 32 & $0,0 \%$ \\
\hline Insuficientemente especificado & 29 & $0,0 \%$ \\
\hline Huichol & 21 & $0,0 \%$ \\
\hline Tojolabal & 16 & $0,0 \%$ \\
\hline Popoloca & 15 & $0,0 \%$ \\
\hline
\end{tabular}




\section{Cont... Tabla 1}

\begin{tabular}{lcc}
\hline Chatino & 10 & $0,0 \%$ \\
Huave & 10 & $0,0 \%$ \\
Otras lenguas América & 8 & $0,0 \%$ \\
Pame & 6 & $0,0 \%$ \\
Matlazintla & 2 & $0,0 \%$ \\
Mayo & 2 & $0,0 \%$ \\
$\begin{array}{l}\text { Q'anjo'al } \\
\text { Total de hablantes de lengua indígena } \\
\text { en el estado }\end{array}$ & 2 & $0,0 \%$ \\
$\begin{array}{l}\text { Población total en el estado de } \\
\text { Hidalgo }\end{array}$ & 606.045 & $21,2 \%$ \\
\hline
\end{tabular}

Fuente: Elaboración propia, 2020 a partir de los datos del Instituto Nacional de los Pueblos Indígenas (2016).

Los Nahuas hablantes, se concentran en la parte norte del territorio del estado de Hidalgo-México, sin embargo, el municipio de Acaxochitlán donde hablan náhuatl, se ubica en la parte sureste de la entidad donde predomina el habla otomí. Hasta el año 2015, la población total acaxochitlense se cuantificó en 43.774 habitantes (INEGI, 2016) lo que representó el 1,53\% de la población total del estado de Hidalgo-México. Sus habitantes mantienen el uso cotidiano de la lengua náhuatl para comunicarse; por lo menos el $36,04 \%$ reconoció ser hablante de una lengua originaria.

Respecto a las condiciones sociales imperantes en el municipio, según mediciones oficiales del Consejo Nacional de Evaluación de la Política de Desarrollo Social (CONEVAL, 2016) para el año 2015 , el $75 \%$ de su población vivía en condición de pobreza, la situación más grave lo enfrentaba el $26,1 \%$ de la población total que fue reconocida en su condición de pobreza extrema, es decir, personas cuya totalidad de ingresos no les alcanza para obtener la alimentación que asegure una vida sana (Anaya, Buelvas y Romero, 2020). Por tanto, en el municipio de Acaxochitlán se concentran dos condiciones: Ruralidad y pobreza, lo que impacta directamente en las vidas y condiciones femeninas.

\section{Metodología}

La estrategia metodológica se estableció con dos necesidades, la primera, porque una instancia del gobierno municipal de Acaxochitlán requería del estudio diagnóstico sobre las mujeres del municipio para revitalizar la política y recursos públicos, lo que significó identificar a los grupos poblacionales según los marcos normativos existentes: Niñas (menores de 12 años), adolescentes (12-18 años), adultas (mujeres menores de 70 años) y adultas mayores (mujeres mayores de 70 años). La segunda necesidad académica, fue documentar las formas culturales en que se construye la desigualdad por razones de género al interior de las comunidades Nahuas; ambos intereses representados por una funcionaria municipal y dos académicas, se pusieron en diálogo para establecer puntos de acuerdo y colaboración que rebasara los constructos ya conocidos de los pueblos indígenas para establecer temas y preguntas según la realidad por conocer y las necesidades del conocimiento (Canales, 2018). 
La investigación es de carácter descriptivo e interpretativo, lo que significó la aplicación de metodología mixta. Los datos y procesamiento cuantitativo, fue para ilustrar condiciones sociales y poblacionales más allá de las comunidades Nahuas, para ello se consultaron bases de datos de los censos de población y encuestas poblacionales generadas por la institución más importante en la creación de información estadística en México que es el Instituto Nacional de Estadística y Geografía (INEGI, 2011) y los Indicadores socioeconómicos de los pueblos indígenas de México generados por la instancia nacional a cargo de la política pública para dicha población, el Instituto Nacional de los Pueblos Indígenas (INPI, 2016).

La técnica cualitativa de grupos de discusión posibilitó la convocatoria de las mujeres, el trabajo operativo de asegurar la asistencia de las participantes quedó a cargo de la Instancia de la Mujer del gobierno municipal de Acaxochitlán, los grupos de discusión tuvieron como ejes de diálogo los derechos a: La igualdad, educación y una vida libre de violencia. El total de las participantes de las 5 comunidades fue de 255. Por comunidad asistieron de Santa Ana Zacuala, 46; Santiago Tepepa, 56; Los Reyes, 46; San Pedro Tlachichilco, 57 y de San Mateo, 50. Cada mujer fue situada en el grupo de sus contemporáneas: Niñas, adolescentes, adultas y adultas mayores. Tal división se condujo según la premisa de grupos etarios que desde la política pública se les asigna recursos para efecto del ejercicio y garantía de los derechos humanos.

Todos los grupos de discusión se desarrollaron en las aulas escolares de las comunidades, el lugar se determinó por tres razones: Tener espacio suficiente y separado entre los grupos específicos; ocupar un lugar conocido y propio de la presencia colectiva femenina; y, eliminar cualquier sospecha masculina sobre la congregación de las mujeres; la mejor opción resultó la escuela primaria donde acuden sus hijos.

Las ideas y las experiencias personales fueron compartidas de manera más fluida en la medida que en el grupo todas eran conocidas y no emparentadas, caso contrario ocurrió cuando coincidían más de dos personas integrantes de una misma familia, porque se aplicaron la vigilancia mutua, situación ya prevista desde la experiencia revisada en otras investigaciones (Arias, 2014). El levantamiento de información, se realizó en los meses de junio y julio del año 2017.

\section{La construcción de la desigualdad de género}

La desigualdad de género, es una condición social que se ha visibilizado para la etapa adulta de la vida de las mujeres, pero es una construcción social cultivada en las familias y desde la infancia, de tal manera que las mujeres en su edad adulta, ya tienen incorporado en su imaginario de género su condición de subordinación frente a los varones, primero aprendieron en relación con sus hermanos, luego observaron la condición de sus madres frente a sus progenitores, para después con sus maridos reproducir el sometimiento de su propia persona y de sus hijas.

No se pretende ignorar la prevalencia de condiciones estructurales del orden social y económico del país, que impactan de manera negativa en los resquicios de las vidas indígenas y no indígenas, especialmente de los sectores históricamente vulnerables: Indígenas, mujeres, discapacitados, huérfanos, entre otros; en una investigación que indagó sobre la pobreza infantil, se demostró que el género y grupo étnico son condiciones sociales que agudizan la pobreza en los hogares, incluso al interior de los mismos, las mujeres, niños y niñas tienen mayor dificultad para acceder a los bienes del hogar (Laverde, Gómez y Sellamén, 2020).

Esta reflexión revisa las particularidades sociales y culturales que hacen posible la vida comunitaria que reproducen la desigualdad de las mujeres; en otras palabras, se sostiene que las comunidades Nahuas de Acaxochitlán, la pervivencia de la vida comunitaria y la 
cultura indígena, están cimentadas en formas patriarcales de dominación, que agreden los derechos de las mujeres desde su infancia.

Tal supuesto conduce a revisar los contenidos, símbolos y significaciones que Nahuas de Acaxochitlán tienen sobre el concepto de persona, lo que significa una separación de las visiones esencialistas que presentan a los pueblos indígenas ajenos de la discriminación y la violencia de género en su vida comunitaria. La dominación por razones de género, es un proceso de raíces profundas, por ello, se ubica al proceso de colonización iniciado en el siglo XVI, que significó la edificación de formas de dominación capitalista, instalación del pensamiento cristiano y la apropiación del racismo, para justificar el acceso desigual de los derechos (Quijano, 2014), necesariamente el sistema colonial impactó a los pueblos indígenas en sus referentes simbólicos y significaciones, especialmente con respecto a las mujeres.

Desde pequeñas, ellas atestiguan, escuchan y viven los mandatos de género que conllevan a la división sexual del trabajo, pero sobre todo un acceso desigual a los recursos materiales y simbólicos de sus familias, un ejemplo ocurrido en la vida diaria es el recurso "tiempo de ocio", que en la infancia está condicionado a que las niñas concluyan las labores domésticas que apoyan el trabajo de la madre, para luego tener ratos de juego o mirar programas de televisión, "mi mamá después de comer siempre nos pone a hacer quehacer o trabajar y los niños se quedan en sus sillas viendo la tele" (Niña de la comunidad de San Mateo).

En las comunidades rurales y agrícolas, hombres y mujeres trabajan de manera intensa, pero el uso del tiempo de ocio es un derecho casi exclusivo de los varones, porque el ocio significa entretenimiento (ver televisión), consumo (ingerir bebidas alcohólicas) o recreación con amigos fuera de la vivienda, esto último conlleva a disipar recursos económicos de la familia que compromete el gasto familiar; así, a través de los padres, como de sus madres, las niñas aprenden que los varones no cumplen siempre con la manutención de sus hogares.

En ese sentido, en las comunidades Nahuas, las niñas aprenden la realización de las labores domésticas, pero sobre todo asumen que sus congéneres varones tienen derechos sobre la disposición de los bienes materiales y simbólicos, ellas prácticamente solo son usuarias de esos bienes, pero nunca titulares incluso de sus propios recursos como es su cuerpo y su tiempo, porque al crecer deben cumplir con el mandato de género, que es vivir en pareja para tener descendencia. Las excepciones, refieren a mujeres solteras que permanecen en la casa paterna, trabajando o realizando labores remuneradas para cubrir sus propias necesidades y cumpliendo con el cuidado de sus progenitores, luego del fallecimiento de sus progenitores, la situación de las solteras es incierta porque quedan sujetas a la voluntad del hermano y cuñada convertidos en titulares de la casa paterna.

Los matrimonios, obedecen a una práctica endogámica validada por las formas de organización comunitaria, que se traducen en vigilancia y castigo para varones ajenos a la comunidad que se atreven a cortejar a las jóvenes; las mujeres que logran matrimonios exogámicos, pierden sus derechos sobre los recursos comunitarios para adscribirse a los derechos del marido en su comunidad de origen. Llegado el momento, las jóvenes pasan de la casa paterna a la vida marital y trabajo doméstico con la familia de su marido, también aprenden que todo lo que hagan y signifiquen ingresos monetarios son extensiones de sus deberes como mujeres cuidadoras de sus familias.

Sus progenitoras saben que sus hijas tendrán reconocimiento cuando se conviertan en madres, así el recurso cuerpo femenino para la reproducción les posibilitará la única propiedad que les reconocerán como mujeres: Los hijos. Por eso, madres, abuelas y todas las mujeres adultas con algún parentesco sobre las jóvenes, fungen como cerco vigilante del cumplimiento de los mandatos de género, incluyendo la violencia de las adultas mayores para las jóvenes que llegan a la vivienda familiar como nueras. 
Los hombres desde temprano se involucran en el trabajo productivo para su futuro papel como abastecedores y vigilantes de sus hogares, en pueblos rurales y agrícolas, los bienes más importantes son la tierra, los insumos para el trabajo y la vivienda. Ello justifica que la tierra productiva y la casa paterna sean derechos masculinos, situación reforzada desde la organización comunitaria, porque la convocatoria y asistencia a las asambleas generales, es un derecho del hombre de la casa; asistir a la asamblea posibilita que los varones salgan electos como autoridades y responsables de comités; la presencia femenina ocurre en calidad de representantes de sus maridos, eso solo les otorga asistencia, pero carecen de voz y voto para la toma de decisiones del interés común.

Al respecto, se identificaron a mujeres titulares de algunos comités: Salud y adultos mayores; su responsabilidad y titularidad se fundamenta en la operatividad de los programas públicos financiados por el Estado mexicano. A diferencia de otras regiones indígenas, donde las mujeres tienen la titularidad de la representación de la escuela a la que acuden sus hijos, como las comunidades contenidas en esta reflexión; los hombres ocupan los cargos más importantes, todo lo cual confirma que persiste una división sexual de los roles y espacios, que obedece a una cultura patriarcal que se mantiene en cualquier espacio que se considere público y externo a la vida familiar.

En ese sentido, Orozco, Jiménez y Cudris-Torres (2020), sostienen que: "Las creencias y mitos culturales asociados al sistema patriarcal han legitimado desde tiempos remotos el poder y la dominación del marido hacia la mujer y los hijos, despojando a éstos de todo derecho legal, económico o social" (p.60).

El resguardo de las mujeres de todas las edades, dificulta que ellas puedan cursar la escuela fuera de la comunidad donde no llega la vigilancia del patriarca, así que las jóvenes saben que su escolaridad solo alcanzará el último año de la escuela localizada en la comunidad, que es la secundaria, lo que suma un total de nueve años de escolaridad, hecho que se cumple en las generaciones menores de treinta años (ver Gráfico I). Aunque tampoco los varones en general se interesan por continuar con estudios de nivel medio superior, porque el mandato de género asociado al trabajo, los presiona para su pronta incorporación total a la actividad económica de la familia.

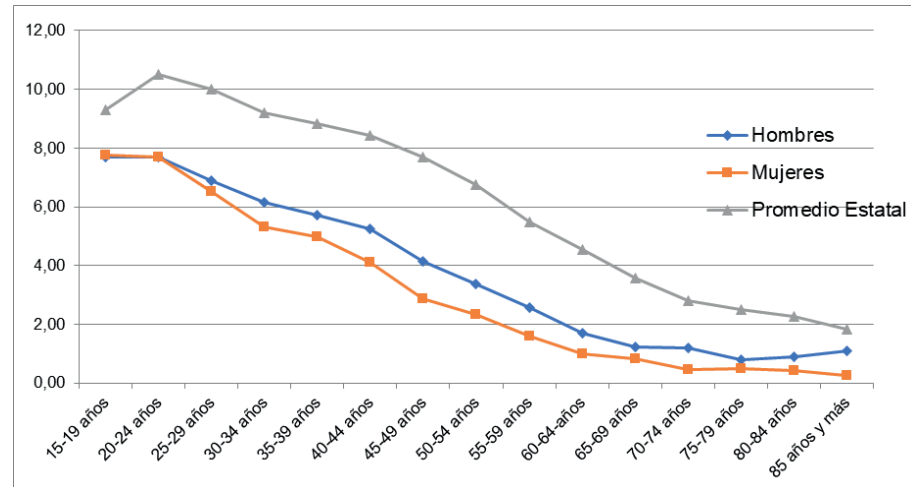

Fuente: Elaboración propia, 2020 con datos del Censo de Población y Vivienda 2010 (INEGI, 2011).

Gráfico 1: Municipio de Acaxochitlán, Hidalgo. Promedio de escolaridad por grupos quinquenales de edad por sexo, 2010 


\section{La violencia en el espacio privado y público}

En otros contextos rurales e indígenas, la posibilidad de lo privado y lo público como ámbitos que distinguen las esferas femeninas así como masculinas pueden ser visibles y tangibles, lo que resulta imposible para los ámbitos indígenas de Acaxochitlán, porque la comunidad al igual que los espacios no obedecen a una estricta división y especialización de usos y finalidades; tampoco los temas y problemas son exclusivos de personas y sus familias, porque los tejidos sociales se sobreponen a manera de niveles, de tal forma que la primera capa refiere a los parentescos consanguíneos, luego se localizan los parentescos por afinidad y rituales, que tienen como núcleo de cohesión: La lealtad para la parentela.

Los otros niveles del tejido social, se sustentan en las necesarias relaciones de colaboración que imponen el cumplimiento de obligaciones comunitarias, por ejemplo: La edificación de obra pública, las fiestas patronales, los apoyos para el cumplimiento de los cargos civiles, la ayuda para la realización de los cargos religiosos, la colaboración para el trabajo en el campo, entre otros.

Por tanto, en contextos indígenas, la vigilancia de los mandatos de género es prerrogativa de la familia y la comunidad, entre ellas no hay una línea clara que distinga los respectivos ámbitos, hecho documentado en mujeres zapatistas a partir de la realización de grupos focales, donde expresaron que los hombres asumían su derecho de golpear a las mujeres, situación que ellas reprobaban en dos sentidos, con los hombres en particular y con las comunidades como colectivo que dictamina el bien común (Millán 2014).

En la lógica organizativa Nahua, se distingue la separación del trabajo comunitario según distinciones sexuales y de género. Para ilustrar lo anterior, se debe recordar la práctica matrimonial endogámica ya referida; la unión de una pareja, involucra a dos grupos familiares que en lo sucesivo tomarán partida de los altibajos de los cónyuges.
En la vida de las parejas, ocurren hechos de violencia física que son expuestos a la sanción de las autoridades locales e incluso de las municipales, pero en el imaginario masculino, la violencia física en los matrimonios no es un hecho grave, porque en el mejor de los casos les imponen una sanción económica para luego regresarlos a sus hogares.

(...) así pasó con mi papá. Muchos años le pegó a mi mamá y no tiene mucho que se separaron. Mi mamá estaba toda ensangrentada de que mi papá le había pegado y le habló a los policías. Los policías llegaron a casa de mi mamá y mi mamá estaba toda golpeada ensangrentada y mi papá, o sea, sucio de su camisa y lo único que dijeron los policías -Es que es Don Beto, ¿cómo nos lo vamos a llevar? ¡Los policías de presidencia toman con mi papá! ¡Es que no! ¡Es que es Don Beto! ¡No se lo llevaron! (Mujer joven, comunidad de Santa Ana Tzacuala).

Resulta común que los infractores de la paz de sus familias tengan estrechos vínculos de parentesco o amistad con los representantes de las comunidades, por tanto, la sanción queda en una represión verbal con una débil promesa de no reincidencia.

Con lo anterior, se busca ilustrar que la dicotomía de lo público y privado en la construcción de los roles de género, queda desdibujada en las comunidades indígenas porque hombres y mujeres transitan según los roles asignados en la intensa vida comunitaria de inspiración patriarcal; las alteraciones del orden establecido en asuntos considerados como de la comunidad, ocurren cuando se insertan actores ajenos o externos, un ejemplo es la operación de las convocatorias y programas de las agencias del gobierno en sus distintos niveles, porque instalan comités de salud, adultos mayores, entre otros, según los beneficios públicos para la comunidad, entonces la participación femenina resulta indispensable pero su incorporación invariablemente pasa por la aprobación de las autoridades locales.

Por tanto, las situaciones que involucran problemas de violencia considerados "domésticos", son atendidos por las 
autoridades que en su calidad de hombres y reconociendo al infractor como su homólogo de género, remiten el problema al hogar y la familia involucrada. La posibilidad de las mujeres agredidas para acudir a las instancias municipales, conlleva trasladarse a la cabecera municipal, lo que involucra tiempo y recursos económicos, pero, dinero y horas para sí mismas es ajeno a la construcción cultural femenina náhuatl, porque su tiempo, trabajo y cuerpo están al servicio de su patriarca, cuya vigilancia es apoyada por hombres y mujeres de la comunidad.

Las jóvenes y niñas asumen que los espacios públicos les impone riesgos, porque enfrentan el acecho masculino que se expresa verbalmente, en asedios que amenazan con tocarlas, y en el peor de los casos, en agredirlas sexualmente. Tales agresiones no son discutidas o revisadas como problemas que competen a la comunidad en las asambleas generales; la omisión es un hecho dado porque el grueso de integrantes de la asamblea son varones, y las mujeres están en representación del varón de su casa, además, en la construcción de la masculinidad local, lo varones asumen como su derecho el asedio de mujeres jóvenes y solteras que transitan en la vía pública.

Lo anterior, tiene una mirada colonizadora que también transformó la sexualidad, de una construcción relacionada con la fecundidad de la tierra a una mirada pornográfica y antropocéntrica, el cual reduce el cuerpo de las mujeres a objeto de deseo y propiedad, pero al mismo tiempo le dota de significado de pecado. Estos hechos han permitido encubrir desde la cultura, la violencia y desigualdades que sufren las mujeres (Segato, 2014; Orozco, et al. 2020).

Para el caso de mujeres casadas o que viven en pareja, son excluidas del acoso, porque su marido tendría la obligación y derecho de reclamar el mantenimiento del orden y respeto sobre los bienes masculinos. Estos hechos coinciden con la afirmación feminista sobre la complicidad masculina como una actitud que mantiene inalteradas las relaciones desiguales entre hombres y mujeres en cualquier contexto, incluso en las comunidades Nahuas.

\section{Conclusiones}

La construcción de persona con derechos y la salvaguarda de los mismos en comunidades indígenas Nahuas está anclada a la condición masculina, en tanto es ajena a las mujeres, a su vez, ambos están sujetos al sentido de propiedad y vigilancia que impone la familia y la comunidad. Los varones rinden cuentas y quedan sujetos a la aprobación de otros hombres, las mujeres quedan sujetas a otras mujeres con mayor jerarquía familiar y a los varones de la familia, sin embargo, el reconocimiento de los derechos femeninos, está anclado al patriarca que la subordina y exige culto.

En el hogar paterno, el culto al patriarca se aprende en la realización de las labores domésticas y la incorporación de la figura masculina como tomador de decisiones sobre las personas y las cosas del hogar. En el ámbito de la vida comunitaria, el culto al patriarca se cultiva porque las mujeres carecen de representación en las instituciones comunitarias donde se toman las decisiones que afectan la vida de la comunidad. Las pocas mujeres participantes en la vida comunitaria se ocupan de las responsabilidades que son vistas como la extensión de su trabajo doméstico y también porque son comités impulsados por instituciones de gobierno para el desarrollo de la política pública. La integración y funcionamiento de los comités son observados por las autoridades locales conformado por hombres.

Las autoridades locales indígenas corresponden a los constructos culturales que asumen como sus pares a los otros hombres, por tanto, el orden social y la toma de decisiones está en razón del rendimiento de cuentas a los otros hombres. Por lo anterior, las mujeres indígenas continúan indefensas en el ejercicio de sus derechos humanos, a pesar de que existen instituciones y políticas públicas, también están permeadas por alianzas 
patriarcales.

Por tanto, el sistema patriarcal de dominación persistente en las comunidades Nahuas no es distinto al instalado en sociedades no étnicas, pero la particularidad está en que la construcción de la persona sujeta de derechos, es una condición anclada en la construcción del individuo con reconocimiento de los otros hombres de la comunidad, las mujeres no tienen reconocimiento individual, porque en realidad son propiedad colectiva de y para sus familias, que les impide construirse como personas individuales con reconocimiento, primero de sus familias y luego de la comunidad. Queda como línea de reflexión, que los derechos desde la lógica indígena se construyen para sus integrantes, especialmente para las mujeres.

\section{Referencias bibliográficas}

Anaya, A. R., Buelvas, J. A., y Romero, Y. (2020). Pobreza e inclusión financiera en el municipio de Montería, Colombia. Revista de Ciencias Sociales (Ve), XXVI(1), 128-143. https://doi. org/10.31876/rcs.v26i1.31315

Arias, P. (2014). La etnografía y la perspectiva de género: nociones y escenarios en debate. En C. Oehmichen (Ed.), La etnografia y el trabajo de campo en las ciencias sociales (pp. 173-194). México: UNAM-IIA

Cabnal, L. (2010). Acercamiento a la construcción de la propuesta de pensamiento epistémico de las mujeres indígenas feministas comunitarias de Abya Yala. En Asociación para la cooperación con el Sur (Eds.), Feministas por siempre. Feminismos diversos: el feminismo comunitario (pp. 11-25). ACSUR.

Canales, M. (2018). Antes del método: Del sentido de la investigación social y el origen de sus preguntas. Cinta de Moebio. Revista de Epistemología de
Ciencias Sociales, (62), 213-220.

Comisión Nacional de los Derechos Humanos - CNDH (2015). Derechos humanos de las mujeres indigenas. CNDH. https:// www.cndh.org.mx/sites/default/files/ documentos/2019-05/Cuaderno Var 34.pdf

Consejo Nacional de Evaluación de la Política de Desarrollo Social - CONEVAL (2016). Medición de la pobreza, Estados Unidos Mexicanos, 20102015. CONEVAL. https://www. coneval.org. $\mathrm{mx} /$ Medicion/Paginas/ Pobreza-municipal.aspx

Gil, J. (1992). La metodología de investigación mediante grupos de discusión. Enseñanza \& Teaching: Revista Interunivesitaria de Didáctica, (1011), 199-214.

Instituto Nacional de Estadística y Geografía - INEGI (2011). México - Censo de Población y Vivienda 2010. INEGI. http://www.inegi.org.mx/rnm/index. php/catalog/71

Instituto Nacional de Estadística y Geografía - INEGI (2016). Panorama sociodemográfico de Hidalgo 2015. http://internet.contenidos.inegi.org. $\mathrm{mx} /$ contenidos/Productos/prod serv/contenidos/espanol/bvinegi/ productos/nueva estruc/inter censal/ panorama/702825082222.pdf

Instituto Nacional de los Pueblos Indígenas INPI (2016). Estimaciones nacionales por entidad federativa. Indicadores Socioeconómicos de los Pueblos Indígenas de México, 2015. INPI. https://www.gob.mx/inpi/articulos/ indicadores-socioeconomicosde-los-pueblos-indigenas-demexico-2015-116128

Kambel, E-R. (2012). Guía sobre los derechos de la mujer indigena bajo la convención internacional sobre la eliminación de todas las formas de 
discriminación contra la mujer. Forest Peoples Programme.

Laverde, H., Gómez, J. J., y Sellamén, A. (2020). Género y grupo étnico: condicionantes de la pobreza infantil en Colombia. Revista de Ciencias Sociales (Ve), XXVI(2), 69-94. https:// doi.org/10.31876/rcs.v26i2.32425

Llanes, A., Cervantes, M. J., Peña, A. A., y Cruz, J. (2020). Maternidad en legislación mexicana: Una visión desde los derechos laborales de la mujer. Revista de Ciencias Sociales (Ve), XXVI(1), 51-60. https://doi. org/10.31876/rcs.v26i1.31310

Millán, M. (2014). Des-ordenando el género/ ¿Des-centralizando la nación?: El Zapatismo de las mujeres indígenas $y$ sus consecuencias. Universidad Nacional Autónoma de México (UNAM).
Orozco, K., Jiménez, L. K., y Cudris-Torres, L. (2020). Mujeres víctimas de violencia intrafamiliar en el norte de Colombia. Revista de Ciencias Sociales (Ve), XXVI(2), 56-68. https:// doi.org/10.31876/rcs.v26i2.32422

Quijano, A. (2014). Colonialidad del poder, eurocentrismo y América Latina. En A. Quijano (Ed.), Cuestiones $y$ horizontes: De la dependencia histórico-estructural a la colonialidad/ descolonialidad del poder (pp. 777. 832). CLACSO.

Segato R. L. (2014). El sexo y la norma: Frente estatal, patriarcado, desposesión, colonidad. Revista Estudos Feministas, 22(2), 593616. https://doi.org/10.1590/S0104026X2014000200012

Segato, R. L. (2016). La guerra contra las mujeres. Traficantes de sueños. 